\title{
Multi-functionalization of lithographically designed gold nanodisks by plasmon- mediated reduction of aryl diazonium salts
}

I. Tijunelyte ${ }^{\mathrm{a}}$, I. Kherbouche ${ }^{\mathrm{b}}$, S. Gam-Derouich ${ }^{\mathrm{b}}$, M. Nguyen $^{\mathrm{d}}$, N. Lidgi-Guigui ${ }^{\mathrm{a}}$, M. Lamy de la

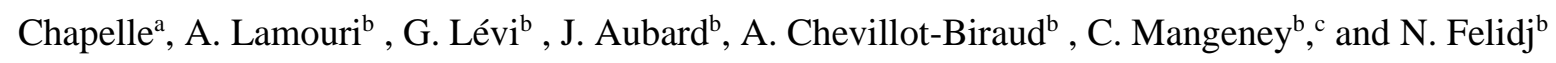

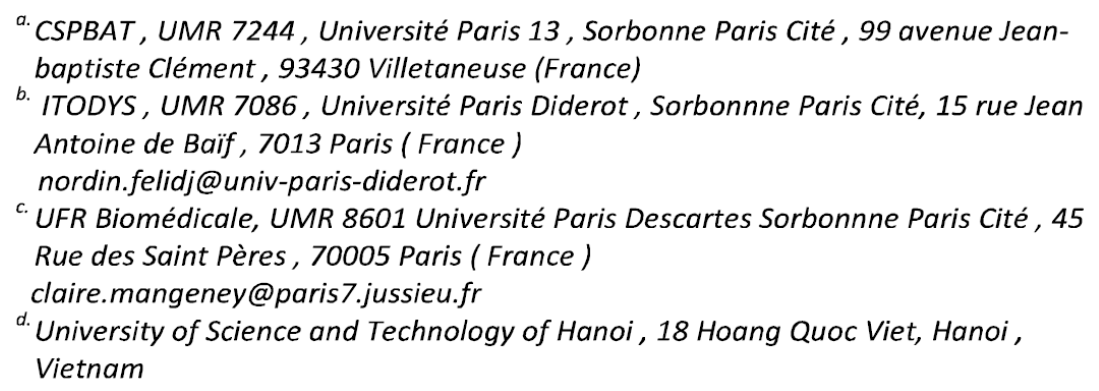

\section{Abstract}

Plasmon-driven surface functionalization of nanoparticles is receiving growing attention as it allows generating locally tailored chemical reactivity on the nanoparticle surface. The extension to surface multi-functionalization still represents a major breakthrough in chemistry. We address this issue by triggering regiospecific surface double-functionalization under plasmon excitation, using diazonium salts as surface functionalization agents. 
Plasmon-driven chemistry has stimulated a wide interest recently since plasmonic nanoparticles (NPs) have been shown to enhance chemical reactions on their surface when illuminated with light of particular frequencies. ${ }^{1}$ These reactions are driven by the excitation of localized surface plasmon resonance (LSPR), resulting in a strong light extinction, a local field enhancement, heat generation, and hot-carrier transfer.1bMany examples of plasmon-driven photochemical reactions were reported including the decomposition of organic molecules, ${ }^{2}$ the oxidative coupling of self-assembled $\mathrm{p}$ aminothiophenol to produce $\mathrm{p}, \mathrm{p}^{\prime}$ - dimercaptoazobenzene, ${ }^{3}$ the dissociation of hydrogen molecules ${ }^{4}$ and the conversion of aldehydes to esters. ${ }^{8}$ Other examples describe the site-selective nanoscale-polymerization of SU8 photoresists on silver nanoparticles, ${ }^{5}$ of pyrrole on gold nanoparticles ${ }^{6}$ or methacrylate derivatives in the inner mesopore walls of mesoporous silica films, ${ }^{7}$ all at room temperature. In the field of surface functionalization, few examples have shown the outstanding perspectives offered by plasmonic NPs to trigger the grafting of organic layers in localized nanoareas. These examples include the photocleavage of self-assembled monolayers close to gold crescents ${ }^{9}$ or the nanoscale regio-selective surface chemistry on gold nanostripes ${ }^{10}$ and silver nanoantennas. ${ }^{11}$ Hot-electron generation was mentioned as the key element for driving reduction reactions. For instance, E. Cortés et al. highlighted a hot-electron reduction reaction of 4-nitrothiophenol on silver nanoantennas with high spatial resolution, with a reactivity depending on the electromagnetic field distribution within the metal. ${ }^{11 a} \mathrm{~N}$. H. Kim et al. observed the reduction of aqueous tetracholoroplatinate ions to zerovalent Pt metal at the surfaces of single gold nanoparticles following the excitation of localized surface plasmon. This reduction and deposition of Pt were attributed to the strong fields, occuring where strong hot electron tunneling currents flow .11b V.-Q. Nguyen et al. recently demonstrated the surface grafting of an organic layer (bisthienylbenzene molecules) triggered by LSP excitation, also attributed to hot electron generation.11c The ability to generate locally tailored chemical reactivity on the nanoparticle surface was further used to guide molecules, ${ }^{12}$ proteins $^{13}$ or quantum dots ${ }^{14}$ to specific regions of the nanoparticle. However, the strategies developed so far provided a single functionalization of the surface with only one type of organic layer grafted in nanoscale region. Extension of these strategies to the multi-functionalization of surfaces represents a major breakthrough in plasmon-mediated chemistry, in order to achieve the grafting of various chemical groups in distinct nanoscale regions.

We address this issue in the present paper by monitoring the incident light polarization on gold nanodisks, to trigger plasmon excitation and obtain regiospecific local surface double-functionalization by diazonium salts (see Scheme 1). Lithographic gold nanodisks, with no capping agents on their surface, were chosen for the proof of concept, as they display a symmetric geometry in the XY plane and a uniform surface chemistry. By playing on the polarization of the incident excitation light, the plasmon-driven functionalization of these isotropic NPs should result in a regioselective surface anisotropy. Functionalization agents based on aryl diazonium salts were selected as they were shown recently to graft on Au nanostripes through plasmon-induced electron transfer. ${ }^{10}$ These 
molecules present several advantages ${ }^{15}$ over self-assembled monolayers derived from thiol molecules: (i) they create strong covalent Au-C bonds with the surface, promoting stable interfacial links between the NPs and the organic functional groups; ${ }^{16}$ (ii) they offer a wide range of terminal functional groups for future post-functionalization reactions. The thickness of the poly(phenylene) layers can be monitored from monolayers to multilayers up to a few tens of nanometers..$^{10}$ In this work, two types of aryl diazonium salts were used, bearing either hydroxyl (4-hydroxyethyl benzene diazonium tetrafluoroborate), or carboxyl-terminated groups (4-carboxy-benzene diazonium tetrafluoroborate). The regioselective grafting of these two types of functional layers, under plasmon excitation, was studied using scanning electron microscopy (SEM), atomic force microscopy (AFM), extinction microspectroscopy and surface-enhanced Raman spectroscopy (SERS). Calculations based on the discrete dipole approximation (DDA) method were also performed to compare the spatial localization of the functional groups around the NPs with the electromagnetic field distribution. This plasmon-mediated multi-functionalization approach opens promising perspectives for the elaboration of nanostructured surfaces with chemical anisotropy.

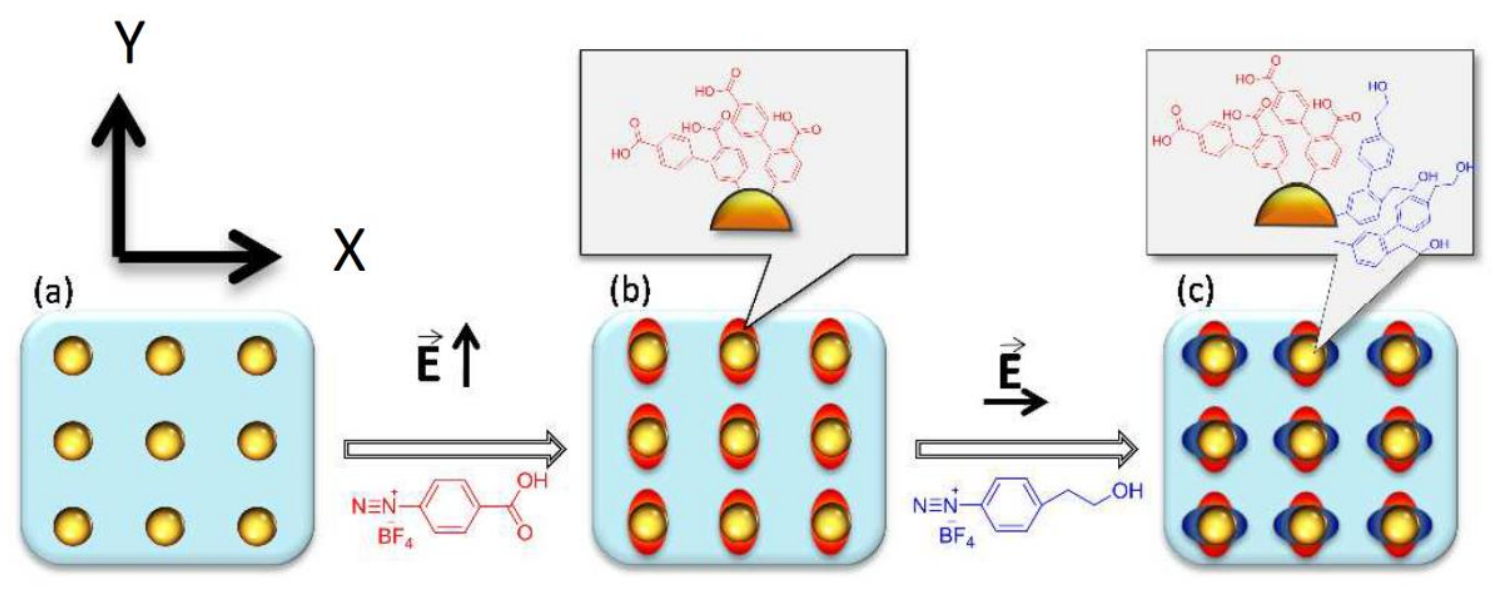

Scheme 1. Scheme of the regioselective multifunctionalization of gold nanodisks under plasmon excitation with polarized light. The nanostructures were first incubated for $180 \mathrm{~s}$ in an aqueous solution of carboxy-phenyl diazonium salt ( $3 \mathrm{mM})$ under laser irradiation polarized in Y direction. Then, the nanostructures were incubated for $180 \mathrm{~s}$ in an aqueous solution containing a hydroxy-ethyl phenyl diazonium salt (3 $\mathrm{mM})$ under laser irradiation, polarized in $\mathrm{X}$ direction.

Lithographic gold nanodisk arrays (diameter $\mathrm{D}=100 \pm 5 \mathrm{~nm}$, height $\mathrm{H}=50 \pm 5 \mathrm{~nm}$, interparticle distance $\Lambda=300 \mathrm{~nm}$ ) were elaborated indium-tin-oxide (ITO) coated-glass substrates by electron-beam lithography (EBL). The fabrication of the samples is detailed in the Supporting 
Information. Representative SEM images and the optical response of the nanodisks are displayed in Figures 1 and S1. The main peak at $655 \mathrm{~nm}$ represents the localized surface plasmon resonance (LSPR) dipolar mode in aqueous solution. Their functionalization by the aryl diazonium salts was first studied in the absence of any plasmon excitation or laser irradiation. After a simple immersion of the plasmonic arrays in aqueous solutions of the diazonium salts at $3 \mathrm{mM}$ during $1 \mathrm{~h}$, a spontaneous grafting of thin poly(aryl) layers all around the nanoparticles was observed. SEM revealed that these layers are uniformly grafted all around the gold nanodisks, with an average thickness of ca. $2 \mathrm{~nm}$. The SERS spectra, displayed in Fig. 2, confirmed the spontaneous grafting of the poly(aryl) layers, with the appearance of two main Raman bands in the range 500-2500 $\mathrm{cm}^{-1}$ (see assignments in Table S1).16
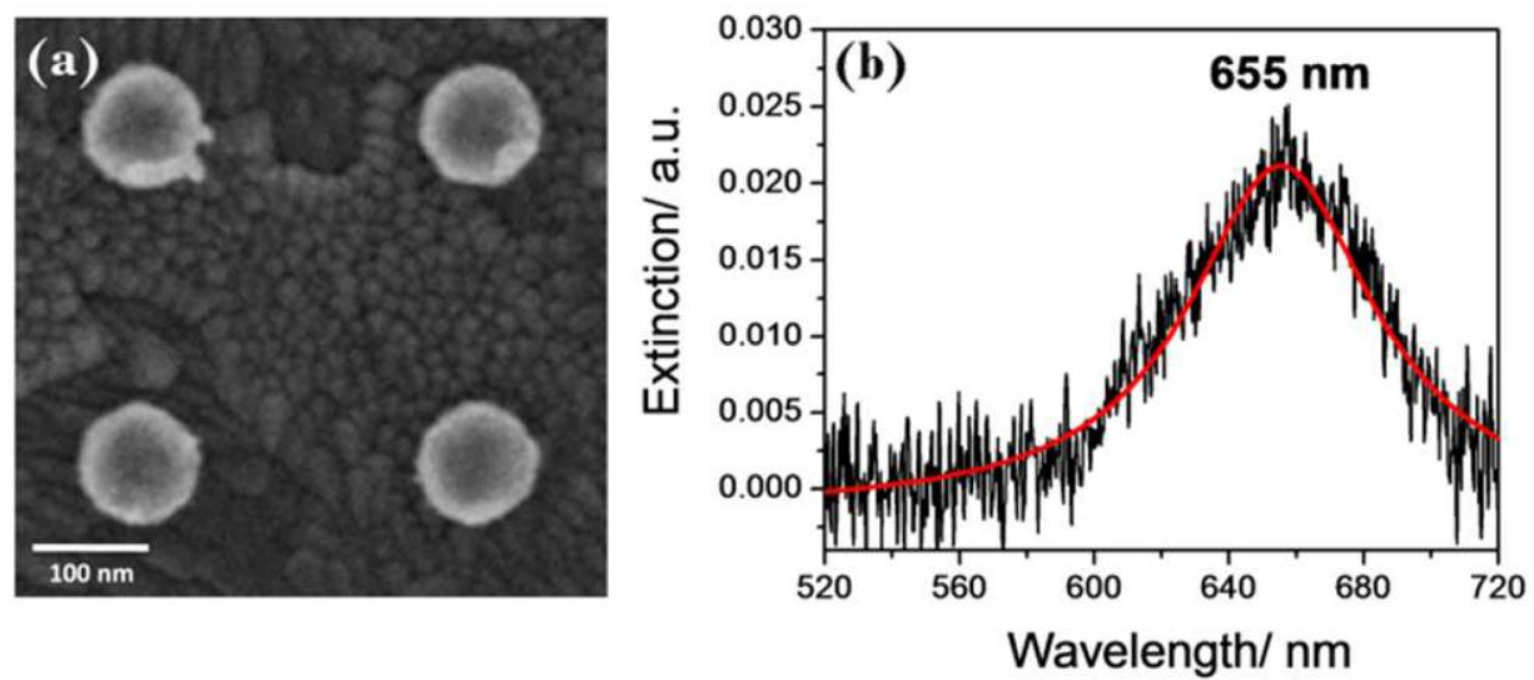

Fig. 1 Characterization of the gold nanodisks array. (a) SEM image of nano-array with disks of D = 100 $\mathrm{nm}$ in diameter. (b) Extinction spectrum in water showing the LSPR of the nanodisks before functionalization.

The most characteristic bands are related to (i) the aromatic ring deformations in the spectral range 1570$1591 \mathrm{~cm}^{-1}$ and (ii) the $\mathrm{CH}$ in-plane bending for mono- and para-substituted benzenes (coupled with ring-N stretching mode) found in the range $1075-1074 \mathrm{~cm}-1$. It is noteworthy that the $\mathrm{N} \equiv \mathrm{N}$ band located around $2300 \mathrm{~cm}-1$, clearly visible in the Raman spectra of the free diazonium salts, disappears upon grafting, confirming the release of $\mathrm{N} 2$ upon reduction of the corresponding diazonium salts. 

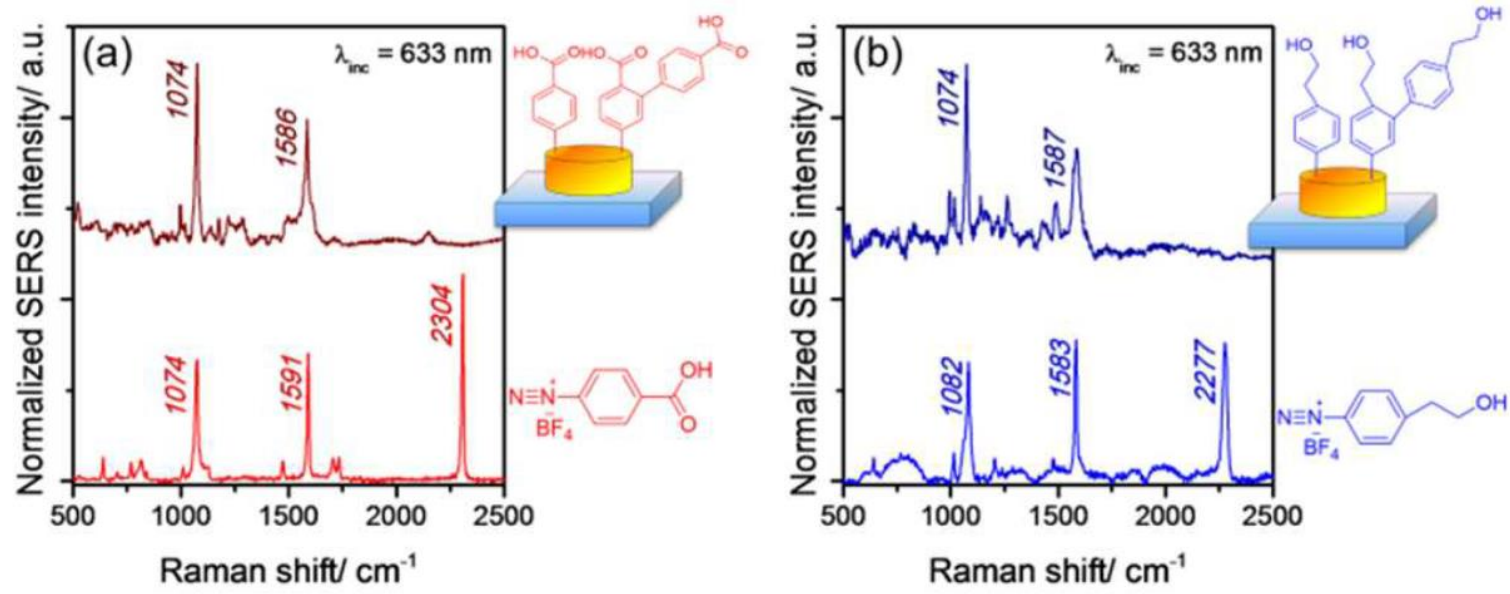

Fig. 2 (a) SERS spectra of the poly(aryl) layers derived from carboxy- or hydoxyethylterminated diazonium salts on the gold nanodisk array after spontaneous grafting. The spontaneous grafting was performed in aqueous solution $(\mathrm{c}=3 \mathrm{mM})$ of the diazonium salt, during 1h. The Raman spectra of the corresponding free diazonium salts are displayed bellow each SERS spectra for the sake of comparison.

In order to demonstrate that the LSPR excitation can locally trigger regiospecific surface functionalization, the gold nanodisk arrays were homogeneously immersed in the solution of carboxyterminated diazonium salts $(\mathrm{c}=3 \mathrm{mM})$ and illuminated by a laser polarized along the $\mathrm{Y}$ axis, during 180 s. The optical exposure was performed under normal incidence, and the He-Ne laser $(\lambda$ inc $=633 \mathrm{~nm})$ was focused on the nanoarray through a microscope objective $(\times 10$ numerical aperture X Y Page 3 of N.A. \#0.25), resulting in a circular laser spot of $\sim 5 \mu \mathrm{m}$ diameter at the surface. The incident wavelength $\lambda$ inc $=633 \mathrm{~nm}$ of the laser excitation matches well the LSPR of the gold nanodisk $(\lambda \mathrm{max}=$ $655 \mathrm{~nm}$ ). It is worth mentioning that laser heating induces the grafting reaction, probably through a cationic pathway, when the exposure energy dose exceeds a given threshold $\mathrm{D}_{\mathrm{th}}$, even in the absence of any plasmon excitation, as shown in a previous paper10. In the case of thermal grafting, the layers are observed all around the nanoparticles, with a uniform distribution. Therefore, an exposure dose below this threshold value, corresponding to $\mathrm{D}=50 \%$ Dth, was used here to avoid side reactions by laser heating and guarantees that the grafting of poly(aryl) layers on the nanostructures was solely induced by LSPR excitation. The plasmon-mediated grafting reaction was shown to induce a red-shift of the plasmon band due to an increase of the local refractive index, from $\lambda \max =655 \mathrm{~nm}$ to $670 \mathrm{~nm}$ (see Fig. S2), revealing the deposition of the organic layers. The obtained hybrid nanostructures were characterized by SEM as the metallic NPs and the poly(aryl) layers show different contrasts (Figure 3a). Polymer lobes can be visualized easily along the $\mathrm{Y}$-axis in the vicinity of the nanodisks. 
In order to verify that the plasmon-induced grafting occurs specifically in the regions of maximum of field enhancement, the mapping of the intensity of the electric field around the nanodisks was calculated by the DDA (see Fig. 3b). It evidences a strong amplification on each side of the nanodisk along the excitation axis with a progressive attenuation away from the axis. From the comparison of the spatial extent of the grafted layers in the XY plane, observed by SEM, it is clear that the regioselective grafting of the poly(aryl) layers is a polymer replica of the dipolar near-field intensity.

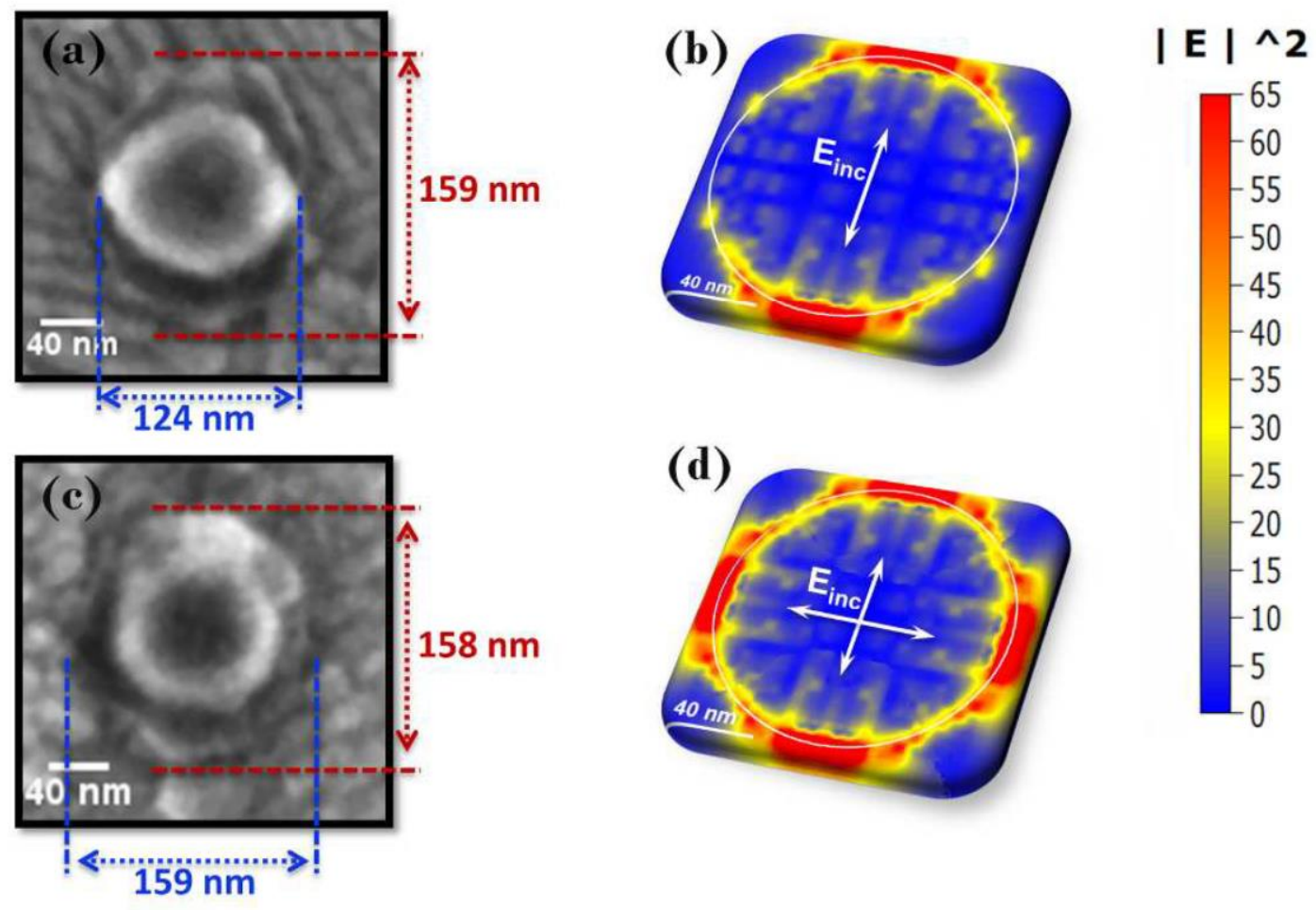

Fig. 3 SEM images of a single nanopartilce after plasmon-induced grafting of (a) carboxyphenyl layers along the $\mathrm{Y}$ direction and (c) additional hydroxyethyl phenyl layers along the $\mathrm{X}$ axis. Irradiation conditions: $\lambda$ inc $=633 \mathrm{~nm}, 180$ seconds with a power of $\mathrm{P}=0.8 \mathrm{~mW} \mu \mathrm{m}-2$. Mapping of the near-field intensity enhancement $|\mathrm{E}| 2$ upon irradiation along the $\mathrm{Y}$ axis (b) and both $\mathrm{X}$ and $\mathrm{Y}$ axis (d), on a unit cell with a nanodisk of $100 \mathrm{~nm}$ diameter, $\mathrm{H}=50 \mathrm{~nm}$, using the DDA method.

The extension of the poly(aryl) layers along the $\mathrm{Z}$ direction (perpendicular to the nanoarray plane) was studied by AFM. The differential AFM profiles of the lateral cross sections (corresponding to the subtraction of the profiles obtained before and after plasmon-induced grafting) revealed no grafting on top of the disk and confirmed the confinement of the grafted layers only in the X-Y plane, on each sides of the nanodisks (see Fig. 4a). The SERS spectra, recorded after plasmon-induced grafting, looked very similar to those obtained after spontaneous reaction apart from an enhancement of the shoulder at 1614 
$\mathrm{cm}^{-1}$, at the higher frequency side of the aromatic ortho-meta $\mathrm{C}=\mathrm{C}$ bond vibration band (Fig. S2c,d). The main Raman band at $1586 \mathrm{~cm}^{-1}$ can be assigned to the aromatic ring deformations in the first layer grafted onto the gold surface, whereas the shoulder at $1614 \mathrm{~cm}^{-1}$ corresponds to the same vibration in the poly(aryl) layer above, covalently linked to the former. The intensity increase of this shoulder thus confirms the polymeric nature of the grafted organic layer. Interestingly, strong variations of the SERS signal intensity were observed depending on the incident polarization. The spectra recorded in the $\mathrm{Y}$ direction, matching the polarization of plasmon-induced grafting, gave the highest SERS signal intensities. Nevertheless, the SERS signal is not fully switched off along the $\mathrm{X}$ direction, perpendicular to the polarization of plasmon-induced grafting. This is due to the spatial extension of the electromagnetic field enhancement away from the irradiation polarization axis leading to a poly(aryl) film, which is not strictly confined along the $\mathrm{Y}$ axis, but slightly spread out towards the $\mathrm{X}$-axis (Fig 3b).
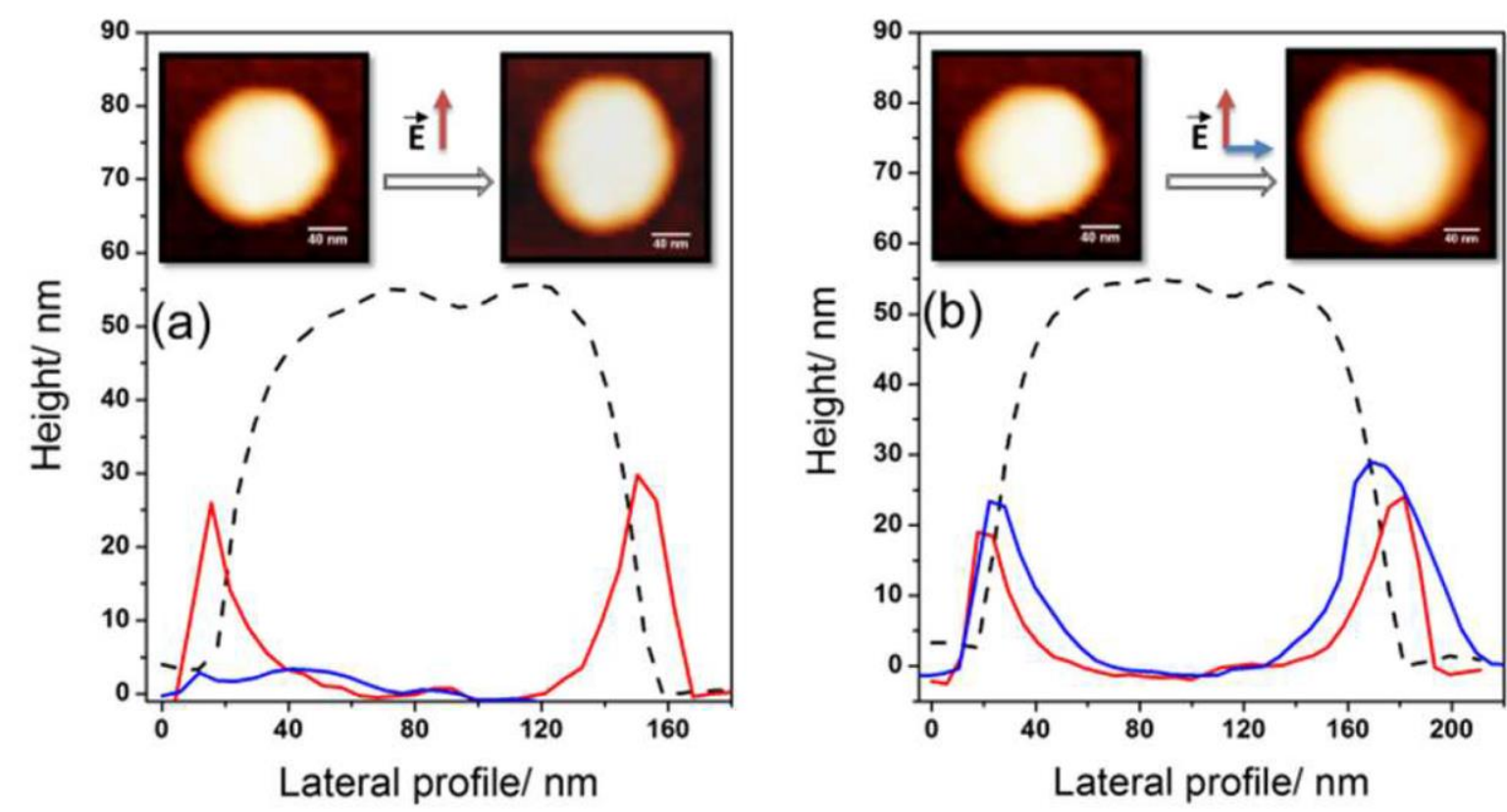

Fig. 4 (a) Plasmon-induced grafting of the first carboxy-aryl layer, using an incident polarization along $\mathrm{Y}$ axis. Top: AFM image of a single disk before (left) and after (right) plasmon-induced grafting along $\mathrm{Y}$ axis. Bottom: Lateral cross section profile along $\mathrm{Y}$ axis after grafting (dashed line) and differential lateral cross section along $\mathrm{Y}$ axis (red line) revealing two main lobes along the $\mathrm{Y}$ direction and along $\mathrm{X}$ (blue line) confirming no grafting along $\mathrm{X}$ direction. (b) Plasmon-induced grafting of the second hydroxyethyl-aryl layer, using an incident polarization along $X$ axis. Top: AFM image of a single disk before grafting (left) and after double plasmon-induced grafting along $\mathrm{Y}$ and $\mathrm{X}$ axis (right). Bottom: Lateral cross section profile along $\mathrm{X}$ axis after grafting (dashed line) and differential lateral cross section along $\mathrm{Y}$ axis (red curve) and $\mathrm{X}$ axis (blue curve) revealing two main lobes along the $\mathrm{Y}$ and $\mathrm{X}$ directions. 
In a second step, the samples were thoroughly cleaned with ethanol and dried before a new immersion in an aqueous solution of the other diazonium salt, hydroxyethyl benzenediazonium. A second exposure with the same energy dose but an incident polarization oriented along the $\mathrm{X}$-axis was then performed. Figure 3c shows the SEM image of the nanodisks after the second grafting step along the $\mathrm{X}$-axis. New polymer nodules are observed on each side of the gold Nanoscale Horizons Page 4 of 12 COMMUNICATION Journal Name 4 | J. Name., 2012, 00, 1-3 This journal is (C) The Royal Society of Chemistry 20xx Please do not adjust margins Please do not adjust margins nanodisks along the $\mathrm{X}$ direction. On the basis of the SEM images recorded at both steps, it turns out that the two different poly(aryl) layers, bearing either carboxyl or hydroxyethyl substituents, were selectively integrated in different orientations around the gold nanodisks via near-field plasmonic excitation.

The AFM differential profile of the single disk before and after the two-step grafting (Fig. 4b) evidences the bi-directional anchoring of the poly(aryl) nanolayers, which perfectly matches the electric field distribution around the nanodisks, according to DDA calculations (Fig. 3d). Note that the corresponding optical and SERS spectra also confirm this bidirectional anchoring, as shown in Fig. S3. The influence of the energy dose upon the spatial extent of the poly(aryl) nodules along the $\mathrm{Y}$ and $\mathrm{X}$ direction was further estimated by SEM. Figure 5 displays the elongation observed in $\mathrm{Y}$ and $\mathrm{X}$ direction. It reveals two important features:

- The growth of the poly(aryl) layer with the energy dose is directional along the excitation polarization axis. Indeed, the elongation along $\mathrm{Y}$ increases with the energy dose when the excitation is polarized along the $\mathrm{Y}$ axis, while the elongation along the $\mathrm{X}$ axis remains constant, and inversely for the $\mathrm{X}$ polarization. The growing of the poly(aryl) layers in $\mathrm{X}$ and $\mathrm{Y}$ directions is also evidenced by extinction spectroscopy, showing a progressive red-shift of the LSP with increased energy dose, and reaching a plateau value at $\sim 1 \mathrm{~mJ} . \mu \mathrm{m}-2$ (Fig. S4).

- The thickness of the grafted layers increases with the energy dose, until reaching ca. 40-60 nm, depending on the diazonium salt: the carboxyphenyl-derived layers appear slightly thicker than the hydroxyethyl ones. 

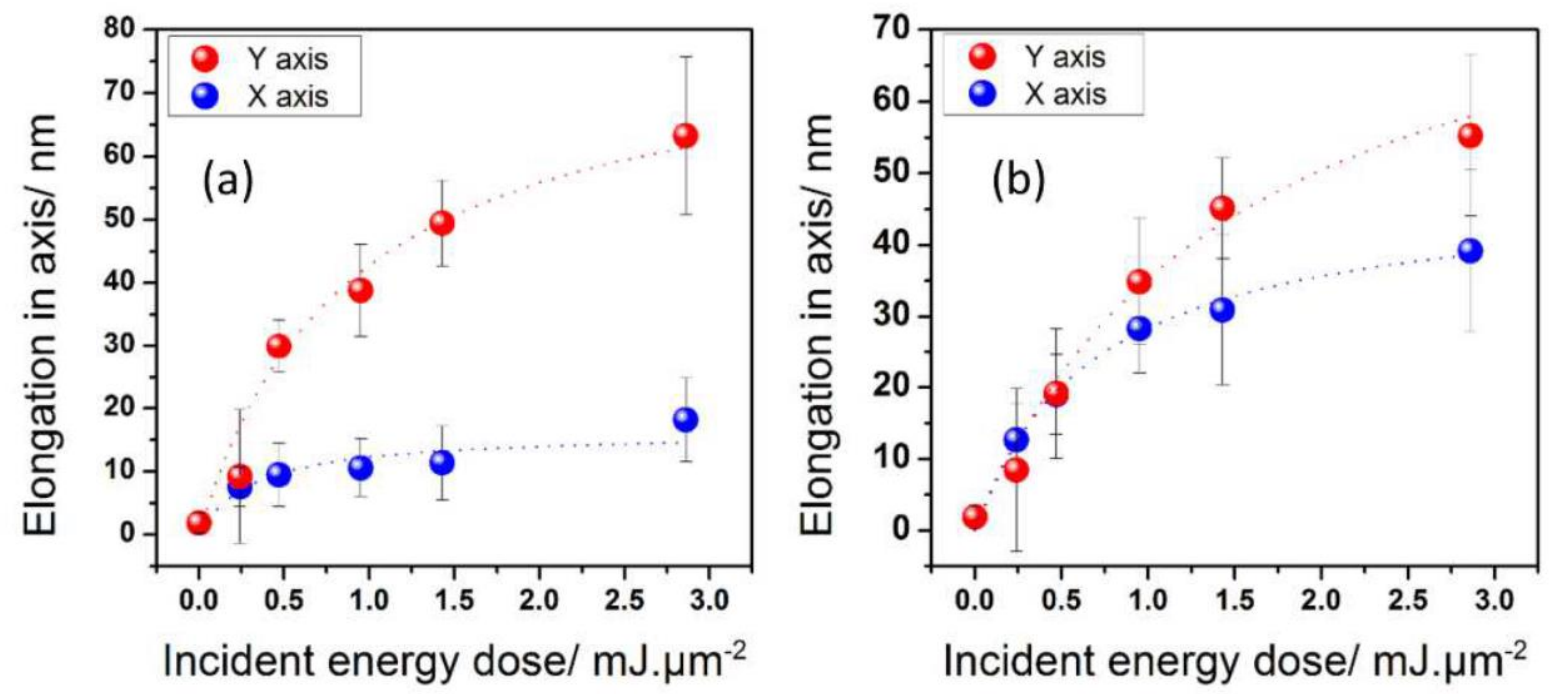

Fig. 5 Plot of the thickness (measured by SEM) of the grafted poly(aryl) layer along the $\mathrm{Y}$ and $\mathrm{X}$ direction, as a function of the incident energy dose from 0 to $3 \mathrm{~mJ} . \mu \mathrm{m}^{2}$, (a) after plasmon-induced first carboxy-aryl layer grafting along $\mathrm{Y}$ and (b) after second hydroxyethyl-aryl layer grafting along $\mathrm{X}$.

The influence of the incident energy dose upon the growth of the carboxy-terminated poly(aryl) layer was also controlled by SERS. The ratio of the relative intensities I1614/I1586 of the two bands at 1614 and $1586 \mathrm{~cm}^{-1}$, assigned respectively to the aromatic ring deformations in the poly(aryl) layer and in the first layer directly grafted on gold via Au-C bonds was plotted against the dose (see Fig.6).
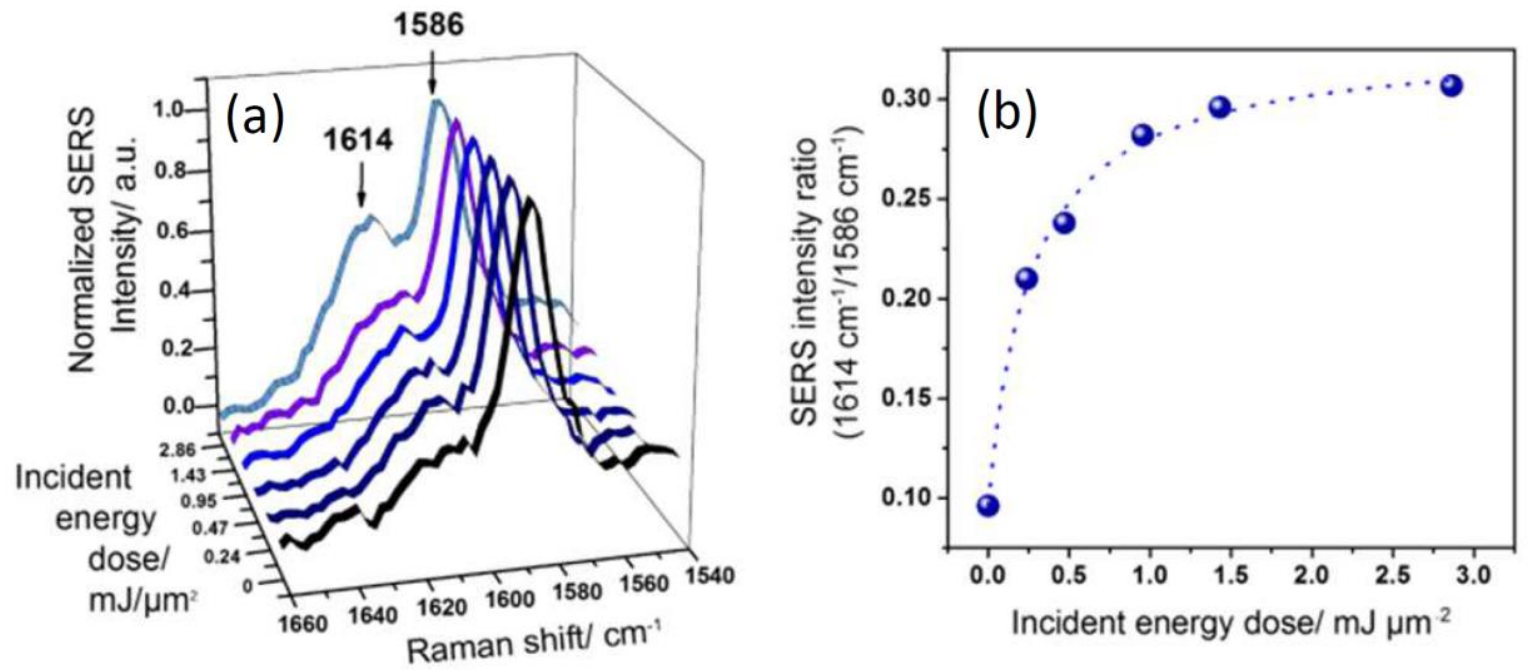
Fig. 6 (a) SERS spectra in the $1540-1660 \mathrm{~cm}^{-1}$ region, recorded on the nanodisk arrays after plasmoninduced grafting of carboxy-phenyl layers along the Y direction; (b) Plot of the I1614/I1586 SERS intensity ratio as a function of the incident energy dose from 0 to $3 \mathrm{~mJ} . \mu \mathrm{m}^{2}$.

An increase in the energy dose leads to a clear enhancement of the shoulder at $1614 \mathrm{~cm}^{-1}$, revealing the growth of the poly(aryl) layer. Although the SEM analysis indicates a continuous thickening of the poly(aryl) layers upon an increase of the incident energy dose, the I1614/I1586 SERS ratio reaches a plateau value at around $1 \mathrm{~mJ} . \mu \mathrm{m}-2$, revealing the maximum distance from the nanostructure's surface probed by SERS. This energy dose corresponds to a poly(aryl) thickness of ca. $40 \mathrm{~nm}$, as shown in Fig. 5 , above which the additional poly(aryl) layers grafted on the gold nanodisks are no longer detected by SERS. Note that this distance correlates perfectly well with the spatial extent of the electromagnetic field, calculated by DDA (see Fig. S5). It is therefore possible to use this plasmonmediated functionalization approach to probe the distance-dependence enhancement effect of SERS in hot spot regions. Moreover, it is an efficient way to modify only the regions of high electromagnetic field whilst leaving the other area of the nanoparticle surface chemically passive.

\section{Conclusions}

In summary, we have shown that it is possible to pattern the surface chemical properties of plasmonic gold nanodisks by two different types of functional poly(aryl) layers, bearing either carboxyl or hydroxyethyl pendant groups. This double functionalization strategy is triggered by the polarization of the incident illumination leading to a site-selective hot-electrons mediated reduction of aryl diazonium salts. This approach offers several advantages over conventional spontaneous grafting methods: (i) it takes place in a few seconds upon laser irradiation, while the usual spontaneous methods require several hours; (ii) it leads to relatively thick poly(aryl) layers (up to 40-60 nm) using low diazonium salt concentrations. Moreover, the thickness of the aryl film can be monitored by the incident light energy. Interestingly, the grafting occurs specifically in the regions of maximum of field enhancement, leaving the other areas of the nanostructure's surface chemically passive. We believe that this plasmonicbased approach will not only pave a new way for the multifunctionalization of plasmonic nanoparticles but also provide a general strategy to attach molecules to hot spot regions and further improve their SERS detection and analysis for (bio) sensing applications.

\section{Notes and references}

1. a) M. Sun, H. Xu, Small 2012, 8, 2777-2786; b) S. Linic, U. Aslam, C. Boerigter, M. Morabito, Nat. Mater. 2015, 14, 567- 576; c) G. Baffou, R. Quidant, Chem. Soc. Rev. 2014, 43, 3898-3907.

2. J. S. Suh, N. H. Jang, D. H. Jeong, M. Moskovits, J. Phys. Chem. 1996, 100, 805-813. 
3. a) Y. F. Huang, H. P. Zhu, G. K. Liu, D. Y. Wu, B. Ren, Z. Q. Tian, J. Am. Chem. Soc. 2010, 132, 9244-9246; b) X. Yan, L. Wang, X. Tan, B. Tian, J. Zhang, Sci. Rep. 2016, 6, 30193.

4. S. Mukherjee, F. Libisch, N. Large, O. Neumann, L. V. Brown, J. Cheng, J. B. Lassiter, E. A. Carter, P. Nordlander, N. J. Halas, Nano Lett. 2013, 13, 240-247.

5. C. Deeb, C. Ecoffet, R. Bachelot, J. Plain, A. Bouhelier, O. Soppera, J. Am. Chem. Soc. 2011, 133, 10535-10542.

6. Y. Takahashi, Y. Furukawa, T. Ishida, S. Yamada, Nanoscale 2016, 8, 8520-8524.

7. N. Herzog, J. Kind, C. Hess, A. Andrieu-Brunsen, Chem. Commun. 2015, 51, 11697-11700.

8. Y. Zhang, Q. Xiao, Y. Bao, Y. Zhang, S. Bottle, S. Sarina, B. Zhaorigetu, H. Zhu, J. Phys. Chem. C 2014, 118, 19062-19069.

9. K.-H. Dostert, M. Álvarez, K. Koynov, A. del Campo, H.-J. Butt, M. Kreiter, Langmuir 2012, 28, 3699-3703.

10. M. Nguyen, A. Lamouri, C. Salameh, G. Levi, J. Grand, L. Boubekeur-Lecaque, C. Mangeney, N. Felidj, Nanoscale 2016, 8, 8633-8640.

11. E. Cortés, W. Xie, J. Cambiasso, A. S. Jermyn, R. Sundararaman, P. Narang, S. Schlücker, S. A. Maier, Nat. Commun. 2017, 8, 14880.

12. Tijunelyte, E. Guenin, N. Lidgi-Guigui, F. Colas, J. Ibrahim, T. Toury, M. Lamy de la Chapelle, Nanoscale 2016, 8, 7105- 7112.

13. B. B. Rajeeva, D. S. Hernandez, M. Wang, E. Perillo, L. Lin, L. Scarabelli, B. Pingali, L. M. LizMarzan, A. K. Dunn, J. B. Shear, Y. Zheng, Adv. Sci. 2015, 2, 1500232.

14. X. Zhou, J. Wenger, F. N. Viscomi, L. Le Cunff, J. Béal, S. Kochtcheev, X. Yang, G. P. Wiederrecht, G. Colas des Francs, A. S. Bisht, S. Jradi, R. Caputo, H. V. Demir, R. D. Schaller, J. Plain, A. Vial, X. W. Sun, R. Bachelot, Nano Lett. 2015, 15, 7458-7466.

15. a) A. A. Mohamed, Z. Salmi, S. A. Dahoumane, A. Mekki, B. Carbonnier, M. M. Chehimi, Adv. Colloid Interface Sci. 2015, 225, 16-36; b) I. Bakas, Z. Salmi, S. Gam-Derouich, M. Jouini, S. Lépinay, B. Carbonnier, A. Khlifi, R. Kalfat, F. Geneste, Y. Yagci, M. M. Chehimi, Surf. Interface Anal. 2014, 46, 1014- 1020; c) M. M. Chehimi, J. Pinson, F. I. Podvorica, Electrode Surface Modification Using Diazonium Salts. Electroanalytical Chemistry; Bard, A.J., Zoski, C.G., Eds.; CRC Press: Boca Raton, FL, 2016, 115-224; d) Y. A. Atmane, L. Sicard, A. Lamouri, J. Pinson, M. Sicard, C. Masson, S. Nowak, P. Decorse, J.-Y. Piquemal, A. Galtayries, C. Mangeney, J. Phys. Chem. C 2013, 117, 26000-26006. 
16. a) L. Laurentius, S. R. Stoyanov, S. Gusarov, A. Kovalenko, R. Du, G. P. Lopinski, M. T. McDermott, ACS Nano 2011, 5, 219-4227; b) S. Betelu, I. Tijunelyte, L. Boubekeur-Lecaque, I. Ignatiadis, J. Ibrahim, S. Gaboreau, C. Berho, T. Toury, E. Guenin, N. Lidgi-Guigui, N. Félidj, E. Rinnert, M. L. d. 1. Chapelle, J. Phys. Chem. C 2016, 120, 18158-18166; c) R. Ahmad, L. Boubekeur-Lecaque, M. Nguyen, S. Lau-Truong, A. Lamouri, P. Decorse, A. Galtayries, J. Pinson, N. Felidj, C. Mangeney, J. Phys. Chem. C 2014, 118, 19098-19105; d) M. Nguyen, X. Sun, E. Lacaze, P. M. Winkler, A. Hohenau, J. R. Krenn, C. Bourdillon, A. Lamouri, J. Grand, G. Lévi, L. Boubekeur-Lecaque, C. Mangeney, N. Félidj, ACS Photonics 2015, 2, 1199-1208. 